International Journal of Applied Mathematics

Volume 33 No. $2 \quad 2020,237-252$

ISSN: $1311-1728$ (printed version); ISSN: 1314-8060 (on-line version)

doi: http://dx.doi.org/10.12732/ijam.v33i2.4

\title{
POISSON AND HEAT SEMIGROUPS FOR THE BESSEL OPERATOR AND ON THE HYPERBOLIC SPACE
}

\author{
Adam Zakria ${ }^{1}$, Ibrahim-Elkhalil Ahmed ${ }^{2}$, Mohamed Vall Ould Moustapha ${ }^{3} \S$ \\ 1,2,3 Department of Mathematics \\ College of Arts and Sciences \\ Gurayat, Jouf University \\ Kingdom of SAUDI ARABIA \\ ${ }^{1}$ Department of Mathematics - Faculty of Sciences \\ University of Kordofan - El-Obeid, SUDAN \\ ${ }^{2}$ Shendi University, Faculty of Sciences and Technology \\ Department of Mathematics, Shendi, SUDAN \\ ${ }^{3}$ Faculté des Sciences et Techniques \\ Université de Nouakchott Al-Aasriya \\ Nouakchott - MAURITANIE
}

\begin{abstract}
In this paper we find explicit formulas for the Poisson and heat semigroups associated to the modified Bessel operator and on the hyperbolic spaces $\mathbb{H}^{n}$.
\end{abstract}

AMS Subject Classification: 35C05, 35C15

Key Words: Poisson semigroup; heat semigroup; Bessel operator; LaplaceBeltrami operator; hyperbolic space

\section{Introduction}

The differential operators of Bessel type and the Laplace-Beltrami operator on the hyperbolic space are known as very important operators in analysis and its applications. This paper deals with the Poisson and heat semigroups as-

Received: August 19, 2019

(c) 2020 Academic Publications

${ }^{\S}$ Correspondence author 
sociated to these second order differential operators. In the last decades the Poisson and heat semigroups associated to many second differential operators have been studied and computed explicitly and there are many interesting papers published in this area of research (see for example Betancor et al. [2], Isolda Cardoso [7], Keles and Bayrakci [15], Stein [16] and the references theirin).

The main objective of this paper is to solve explicitly the following Poisson and heat problems

$$
\begin{gathered}
\left\{\begin{array}{c}
L^{a} u(y, x)=-\frac{\partial^{2}}{\partial y^{2}} u(y, x),(y, x) \in \mathbb{R}^{+} \times \mathbb{R}, \\
u(0, x)=u_{0}(x), u_{0} \in C_{0}^{\infty}\left(\mathbb{R}^{+}\right)
\end{array}\right. \\
\left\{\begin{array}{c}
\mathcal{L}_{n} U(y, w)=-\frac{\partial^{2}}{\partial y^{2}} U(y, w),(y, w) \in \mathbb{R}^{+} \times H^{n}, \\
U(0, w)=U_{0}(w), U_{0} \in C_{0}^{\infty}\left(\mathbb{H}^{n}\right)
\end{array}\right. \\
\left\{\begin{array}{c}
\mathcal{L}_{n} V(t, w)=\frac{\partial}{\partial t} V(t, w),(t, w) \in \mathbb{R}^{+} \times \mathbb{H}^{n}, \\
V(0, w)=V_{0}(w), V_{0} \in C_{0}^{\infty}\left(\mathbb{H}^{n}\right)
\end{array}\right.
\end{gathered}
$$

and

$$
\left\{\begin{array}{c}
L^{a} v(t, x)=\frac{\partial}{\partial t} v(t, x),(t, x) \in \mathbb{R}^{+} \times \mathbb{R} \\
v(0, x)=v_{0}(x), v_{0} \in C_{0}^{\infty}\left(\mathbb{R}^{+}\right)
\end{array},\right.
$$

where

$$
L^{a}=x^{2} \frac{\partial^{2}}{\partial x^{2}}+x \frac{\partial}{\partial x}-a^{2} x^{2}
$$

and

$$
\mathcal{L}_{n}=x_{n}^{2} \Delta_{n-1}+x_{n}^{2} \frac{\partial^{2}}{\partial x_{n}^{2}}+(2-n) x_{n} \frac{\partial}{\partial x_{n}}+\frac{(n-1)^{2}}{4},
$$

are respectively the Bessel operator on $\mathbb{R}^{+}$and the Laplace-Beltrami operator on the half space model of the hyperbolic space $\mathbb{H}^{n}$.

\section{Poisson semigroup associated to Bessel operator}

In this section we give explicit formulas for the Poisson semigroup associated to the Bessel operator $L^{a}$, that is we prove the following theorem. 
Theorem 1. For $a \in \mathbb{R}^{*}$ the Poisson problem (1) has the solution given by

$$
u(y, x)=\int_{0}^{\infty} p_{a}\left(y, x, x^{\prime}\right) u_{0}\left(x^{\prime}\right) \frac{d x^{\prime}}{x^{\prime}}
$$

with

$$
p_{a}\left(y, x, x^{\prime}\right)=\frac{|a|}{\pi} \frac{x x^{\prime} \sin y K_{1}\left(|a| \sqrt{x^{2}+x^{\prime 2}-2 x x^{\prime} \cos y}\right)}{\sqrt{x^{2}+x^{\prime 2}-2 x x^{\prime} \cos y}},
$$

and $K_{1}$ is the modified Bessel functions of second kind.

Proof. To see that the function $u(y, x)$ satisfies the Poisson equation in (1), set $\varphi\left(y, x, x^{\prime}\right)=\phi(z)$, with $z=x^{2}+x^{\prime 2}-2 x x^{\prime} \cos y$, then we have

$$
\frac{\partial \varphi}{\partial x}=\left(2 x-2 x^{\prime} \cos y\right) \frac{\partial \phi}{\partial z}, \quad \frac{\partial^{2} \varphi}{\partial x^{2}}=\left(2 x-2 x^{\prime} \cos y\right)^{2} \frac{\partial^{2} \phi}{\partial z^{2}}+2 \frac{\partial \phi}{\partial z},
$$

and

$$
\frac{\partial \varphi}{\partial y}=2 x x^{\prime} \sin y \frac{\partial \phi}{\partial z}, \quad \frac{\partial^{2} \varphi}{\partial y^{2}}=\left(2 x x^{\prime} \sin y\right)^{2} \frac{\partial^{2} \phi}{\partial z^{2}}+2 x x^{\prime} \cos y \frac{\partial \phi}{\partial z} .
$$

By the above formulas we have

$$
\left(L^{a}+\frac{\partial^{2}}{\partial y^{2}}\right) \varphi=4 x^{2}\left(z \frac{\partial^{2} \phi}{\partial z^{2}}+\frac{\partial \phi}{\partial z}-\frac{a^{2}}{4} \phi\right),
$$

and we see that the first equation in the problem (1) is equivalent to

$$
z^{2} \phi_{z z}+z \phi_{z}-\frac{a^{2}}{4} z \phi=0,
$$

which is a particular case of Lommel differential equation for modified Bessel functions

$$
\left[z^{2} \frac{\partial^{2} \phi}{\partial z^{2}}+(1-2 \alpha) z \frac{\partial \phi}{\partial z}-\left(\beta \gamma z^{\gamma}\right)^{2} \phi+\left(\alpha^{2}-\nu^{2} \gamma^{2}\right) \phi=0,\right.
$$

with $\alpha=0, \nu=0, \beta=1$ and $\gamma=1 / 2$, an approprite solution is $\phi(z)=$ $c K_{0}\left(z^{1 / 2}\right)$, where $K_{0}$ is the modified Bessel function of second kind.

This means that the function

$$
\varphi\left(y, x, x^{\prime}\right)=c K_{0}\left(|a| \sqrt{x^{2}+x^{2}-2 x x^{\prime} \cos y}\right)
$$


satisfies the equations

$$
L_{x}^{a} \varphi\left(y, x, x^{\prime}\right)=L_{x^{\prime}}^{a} \varphi\left(y, x, x^{\prime}\right)=-\frac{\partial^{2}}{\partial y^{2}} \varphi\left(y, x, x^{\prime}\right),
$$

and in consequence it is a solution of the first equation in (1).

By the formula $K_{0}^{\prime}(z)=-K_{1}(z)$ we see that

$$
p_{a}\left(y, x, x^{\prime}\right)=-\frac{1}{\pi} \frac{\partial}{\partial y} \varphi\left(y, x, x^{\prime}\right)
$$

and $p_{a}\left(y, x, x^{\prime}\right)$ satisfies the same equation in (1).

To finish the proof of Theorem 1 it remains to show the limit condition. For this set $z=x^{2}+x^{\prime 2}-2 x x^{\prime} \cos y=2 x x^{\prime}\left(\frac{x^{2}+x^{\prime 2}}{2 x x^{\prime}}-\cos y\right)$ and $x=e^{X}$ and $x^{\prime}=e^{X^{\prime}}$ to obtain:

$$
z=4 e^{X+X^{\prime}}\left\{\sinh ^{2} \frac{\left(X-X^{\prime}\right)}{2}+\sin ^{2}(y / 2)\right\} .
$$

Replacing in (5) we obtain:

$$
u(y, x)=\tilde{u}(y, X)=\int_{0}^{\infty} P_{a}\left(y, X, X^{\prime}\right) \tilde{u}_{0}\left(X^{\prime}\right) d X^{\prime},
$$

with

$$
P_{a}\left(y, X, X^{\prime}\right)=\frac{|a|}{\pi} \frac{e^{\left(X+X^{\prime}\right) / 2} \sin y K_{1}\left(|a| \sqrt{\sinh ^{2} \frac{\left(X-X^{\prime}\right)}{2}+\sin ^{2} \frac{y}{2}}\right)}{\sqrt{\sinh ^{2} \frac{\left(X-X^{\prime}\right)}{2}+\sin ^{2} \frac{y}{2}}} .
$$

Setting $\sinh \frac{\left(X^{\prime}-X\right)}{2}=s \sin y / 2$ or $X^{\prime}=X+2 \arg \sinh (s \sin y / 2)$ we can write

$$
\begin{gathered}
u(y, x)=\tilde{u}(y, X)=\frac{|a|}{\pi} \int_{-\infty}^{\infty} e^{X+\arg \sinh (s \sin y / 2)} \sin y \\
\times \frac{K_{1}\left(2|a| e^{X+\arg \sinh (s \sin y / 2)} \sin (y / 2) \sqrt{1+s^{2}}\right)}{\sqrt{1+s^{2}}} \\
\times \frac{\tilde{u}_{0}(X+\arg \sinh (s \sin y / 2)) 2 d s}{\sqrt{1+s^{2} \sin ^{2}(y / 2)}} .
\end{gathered}
$$

Now we use the asymptotic formula for the modified Bessel function of second kind (Lebedev [9], p.136) $K_{\nu}(z) \sim \frac{2^{\nu-1} \Gamma(\nu)}{z^{\nu}}, z \longrightarrow 0$ we obtain

$$
\lim _{y \longrightarrow 0} u(y, x)=\lim _{y \longrightarrow 0} \tilde{u}(y, X)=\tilde{u}_{0}(X) \frac{1}{\pi} \int_{-\infty}^{\infty} \frac{d s}{1+s^{2}}=\tilde{u}_{0}(X)=u_{0}(x)
$$

and this finishes the proof of Theorem 1 . 


\section{Poisson equation on the hyperbolic space}

In this section we consider the Poisson equation on the hyperbolic upper half space.

Let $\mathbb{H}^{n}=\left\{w=\left(x_{1}, x_{2}, \ldots x_{n}\right) \in \mathbb{R}^{n}, x_{n}>0\right\}$ be the hyperbolic half space endowed with the usual hyperbolic metric

$$
d s^{2}=\frac{d x_{1}^{2}+d x_{2}^{2}+\ldots+d x_{n}^{2}}{x_{n}^{2}},
$$

the metric $d s$ is invariant with respect to the motion group $G=S O(n, 1)$, the hyperbolic volume form $d \mu(w)$ is

$$
d \mu(w)=\frac{d x_{1} d x_{2} \ldots d x_{n}}{x_{n}^{n}},
$$

and the hyperbolic distance $\rho\left(w, w^{\prime}\right)$ given as

$$
\cosh ^{2}\left(\rho\left(w, w^{\prime}\right) / 2\right)=\frac{\left|w-w^{\prime}\right|^{2}}{4 x_{n} x_{n}^{\prime}}+1,
$$

with the Laplace Beltrami operator

$$
\mathcal{L}_{n}=x_{n}^{2} \Delta_{n}+(2-n) \frac{\partial}{\partial x_{n}}+((n-1) / 2)^{2},
$$

where $\Delta_{n}=\sum_{j=1}^{n} \frac{\partial^{2}}{\partial x_{j}^{2}}$ is the Euclidean Laplacian on $\mathbb{R}^{n}$. Before giving the main result of this section we start by the following lemma in which we compute the Fourier transform of the Poisson semigroup $p_{|\xi|}$ for the Bessel operator with respect to the parameter $|\xi|$.

Lemma 2. Set $\xi=\left(\xi_{1}, \xi_{2}, \ldots, \xi_{n-1}\right)$ and $x=\left(x_{1}, x_{2}, \ldots, x_{n-1}\right)$, let $p_{|\xi|}\left(y, x_{n}\right.$, $\left.x_{n}^{\prime}\right)$ be the kernel of the Poisson semigroup for Bessel operator given in (6), then the following formula holds:

$$
\mathcal{F}^{-1}\left[p_{|\xi|}\left(y, x_{n}, x_{n}^{\prime}\right)\right](x)=\frac{2^{(n-1) / 2} \Gamma((n+1) / 2)}{\pi} \frac{x_{n} x_{n}^{\prime} \sin y}{\left(z^{2}+|x|^{2}\right)^{(n+1) / 2}},
$$

with $z=\sqrt{x^{2}+x^{2}-2 x x^{\prime} \cos y}$.

Proof. From the formula giving the Fourier transform of a radial function

$$
\mathcal{F}^{-1}[f](|x|)=|x|^{1-n / 2} \int_{0}^{+\infty} J_{\frac{n-2}{2}}(\rho|x|) f(\rho) \rho^{n / 2} d \rho,
$$


we obtain

$$
\begin{gathered}
\mathcal{F}^{-1}\left[P_{|\xi|}\left(y, x_{n}, x_{n}^{\prime}\right)\right](x)=\frac{x_{n} x_{n}^{\prime} \sin y}{\pi z}|x|^{3-n) / 2} \\
\times \int_{0}^{+\infty} K_{1}(\rho \mid z) J_{\frac{n-3}{2}}(\rho|x|) \rho^{\frac{n+1}{2}} d \rho .
\end{gathered}
$$

By the formula from Prudnikov et al. ([14], p.365)

$$
\int_{0}^{+\infty} x^{\alpha-1} J_{\mu}(b x) K_{\nu}(c x) d x=A_{\mu, \nu}^{\alpha}
$$

where

$$
\begin{gathered}
A_{\mu, \nu}^{\alpha}=2^{\alpha-2} b^{\mu} c^{-(\alpha+\mu)} \frac{\Gamma((\alpha+\mu+\nu) / 2 \Gamma((\alpha+\mu-\nu) / 2)}{\Gamma(\mu+1)} \\
\quad \times{ }_{2} F_{1}\left((\alpha+\mu+\nu) / 2,(\alpha+\mu-\nu) / 2, \mu+1,-\frac{b^{2}}{c^{2}}\right),
\end{gathered}
$$

with $\alpha=(n+3) / 2, \mu=(n-3) / 2, \nu=1, b=|x|, c=z$ we have

$$
\begin{gathered}
\int_{0}^{+\infty} K_{1}(r \mid z) J_{\frac{n-3}{2}}(r|x|) r^{\frac{n+1}{2}} d r=A_{1,(n-3) / 2}^{(n+3) / 2}, \\
A_{1,(n-3) / 2}^{(n+3) / 2}=\frac{|x|^{(3-n) / 2}}{\pi z} 2^{(n-1) / 2} \frac{|x|^{(n-3) / 2}}{z^{n}} \Gamma((n+1) / 2) \\
\quad \times F\left(\left((n+1) / 2,(n-1) / 2(n-1) / 2-\frac{|x|^{2}}{z^{2}}\right) .\right.
\end{gathered}
$$

Now from the formula $F(a, b, b, z)=(1-z)^{-a}$, we obtain the result of Lemma 2 .

Theorem 3. The Poisson problem (2) in hyperbolic space $\mathbb{H}^{n}$ has the solution given by

$$
U(y, w)=\int_{0}^{\infty} P_{n}^{I H}\left(y, w, w^{\prime}\right) U_{0}\left(w^{\prime}\right) d \mu\left(w^{\prime}\right)
$$

with

$$
P_{n}^{I H}\left(y, w, w^{\prime}\right)=\frac{\Gamma((n+1) / 2)}{\pi^{(n+1) / 2}} \frac{\sin y}{\left(2 \cosh d\left(w, w^{\prime}\right)-2 \cos y\right)^{(n+1) / 2}} .
$$


Proof. By the following formula intertwining the Laplace-Beltrami operator $\mathcal{L}_{n}$ on the hyperbolic space $\mathbb{H}^{n}$ and the Bessel operator $L^{|\xi|}$

$$
\mathcal{F}\left[x_{n}^{-(n-1) / 2} \mathcal{L}_{n} x_{n}^{(n-1) / 2} \phi\right](\xi)=L^{|\xi|} \mathcal{F} \phi(\xi),
$$

the Poisson problem on the hyperbolic space (2) is transformed into the BesselPoisson problem (1), with

$$
u\left(y, x_{n}\right)=\mathcal{F}\left[x^{(1-n) / 2} U\left(y, x, x_{n}\right)\right](\xi)
$$

and $u_{0}\left(x_{n}\right)=x_{n}^{(1-n) / 2} \mathcal{F}\left[U_{0}\left(x, x_{n}\right)\right](\xi)$

$$
\begin{gathered}
\mathcal{F}\left[x^{(1-n) / 2} U\left(y, x, x_{n}\right)\right](\xi) \\
=\int_{0}^{\infty} P_{|\xi|}\left(y, x_{n}, x_{n}^{\prime}\right) x_{n}^{\prime(1-n) / 2} \mathcal{F}\left[U_{0}\right]\left(\xi, x_{n}^{\prime}\right) \frac{d x_{n}^{\prime}}{x_{n}^{\prime}}, \\
U\left(y, x, x_{n}\right) \\
=\int_{0}^{\infty} \mathcal{F}^{-1}\left[P_{|\xi|}\left(y, x_{n}, x_{n}^{\prime}\right) x_{n}^{\prime(1-n) / 2} \mathcal{F}\left[U_{0}\right]\left(\xi, x_{n}^{\prime}\right)\right](x) \frac{d x_{n}^{\prime}}{x_{n}^{\prime}}, \\
U\left(y, x, x_{n}\right)=(2 \pi)^{-(n-1) / 2} \int_{0}^{\infty} \mathcal{F}^{-1}\left[P_{|\xi|}\left(y, x_{n}, x_{n}^{\prime}\right)\right](x) \\
\times U_{0}\left(x, x_{n}^{\prime}\right) x_{n}^{\prime(1-n) / 2} \frac{d x_{n}^{\prime}}{x_{n}^{\prime}}, \\
U\left(y, x, x_{n}\right)=(2 \pi)^{-(n-1) / 2} \int_{0}^{\infty} \int_{\mathbb{R}^{n-1}} \mathcal{F}^{-1}\left[P_{|\xi|}\left(y, x_{n}, x_{n}^{\prime}\right)\right]\left(x-x^{\prime}\right) \\
\times U_{0}\left(x^{\prime}, x_{n}^{\prime}\right) x_{n}^{(n-1) / 2} x_{n}^{\prime(n-1) / 2} \frac{d x^{\prime} d x_{n}^{\prime}}{x_{n}^{\prime n}}, \\
U\left(y, x, x_{n}\right)=\int_{\mathbb{H}^{n}} P_{n}^{H}\left(y, w, w^{\prime}\right) u_{0}\left(w^{\prime}\right) d \mu\left(w^{\prime}\right),
\end{gathered}
$$

with

$$
P_{n}^{\mathbb{H}}\left(y, w, w^{\prime}\right)=\frac{\Gamma((n+1) / 2)}{\pi^{(n+1) / 2}} \frac{\sin y}{\left(2 \cosh d\left(w, w^{\prime}\right)-2 \cos y\right)^{(n+1) / 2}},
$$

and the proof of Theorem 3 is finished. 
Proposition 4. Let $P_{n}^{\mathbb{I H}}\left(y, w, w^{\prime}\right)$ be the Poisson kernel on the hyperbolic space $\mathbb{H}^{n}$ then we have:

i) $\left(-\frac{\partial}{2 \pi \sinh \rho \partial \rho}\right) P_{n}^{\mathbb{H}}\left(y, \rho\left(w, w^{\prime}\right)\right)=P_{n+2}^{\mathbb{H}}\left(y, \rho\left(w, w^{\prime}\right)\right)$,

ii) $\int_{r}^{\infty} \frac{P_{n+1}^{\mathbb{H}}(y, \rho)}{\sqrt{\cosh ^{2} \rho / 2-\cosh ^{2} r / 2}} \sinh \rho d \rho=P_{n}^{\mathbb{H}}(y, r)$.

Proof. The part i) is simple, to prove ii) set

$$
\begin{aligned}
& I=\int_{r}^{\infty} C_{n+1} \frac{\sin y}{(\cosh \rho-\cos y)^{(n+2) / 2}}\left(\cosh ^{2} \frac{\rho}{2}-\cosh ^{2} \frac{r}{2}\right)^{\frac{-1}{2}} \sinh r d \rho, \\
& I=\sqrt{2} \int_{r}^{\infty} C_{n+1} \frac{\sin y}{(\cosh \rho-\cos y)^{(n+2) / 2}}(\cosh \rho-\cosh r)^{\frac{-1}{2}} \sinh r d \rho,
\end{aligned}
$$

with $C_{n+1}=\frac{\Gamma(n+2) / 2)}{(2 \pi)^{(n+2) / 2}}$. Set $\cosh \rho=\sigma$ and set $\sigma-\cosh r=\mu$, we see that

$$
\begin{gathered}
I=\sqrt{2} C_{n+1} \int_{\cosh r}^{\infty} \sin y(\sigma-\cos y)^{-(n+2) / 2}(\sigma-\cosh r)^{-1 / 2} d \sigma, \\
I=\sqrt{2} C_{n+1} \sin y \int_{0}^{\infty}(\mu-(\cos y-\cosh r))^{-(n+2) / 2} \mu^{-1 / 2} d \mu,
\end{gathered}
$$

$I=\sqrt{2} C_{n+1} \sin y(\cosh r-\cos y)^{-(n+2) / 2} \times$

$$
\int_{0}^{\infty}\left(1+\frac{1}{(\cosh r-\cos y)} \mu\right)^{-(n+2) / 2} \mu^{-1 / 2} d \mu .
$$

By the formula (Magnus et al. [11], p.13)

$$
\int_{0}^{\infty} t^{x-1}(1+b t)^{-x-y} d t=b^{-x} B(x, y),
$$

where $B$ is the beta function, with $x=1 / 2$ and $y=(n+1) / 2$ we obtain

$$
\begin{gathered}
I=\sqrt{2} C_{n+1} \sin y(\cosh r-\cos y)^{-(n+1) / 2} B(1 / 2,(n+1) / 2), \\
I=\sqrt{2} \frac{\Gamma((n+1) / 2)}{2^{(n+2) / 2} \pi^{(n+1) / 2}} \sin y(\cosh r-\cos y)^{-(n+1) / 2}=P_{n}^{\mathbb{H}}(y, r),
\end{gathered}
$$

thus we obtain ii) and the proof of Proposition 4 is finished. 


\section{Heat semigroup on the hyperbolic space}

In this section we give a new explicit formula for the heat kernel on the hyperbolic space $\mathbb{H}^{n}$.

Proposition 5. Let $e^{-y \sqrt{-\mathcal{L}_{n}}}$ and $e^{t \mathcal{L}_{n}}$ be the Poisson and heat semigroups on the hyperbolic space $\mathbb{H}^{n}$ then we have:

i) $e^{t \mathcal{L}_{n}}=(4 t)^{-1 / 2} L_{y^{2}}^{-1}\left[\frac{\sqrt{\pi} e^{-y \sqrt{-\mathcal{L}_{n}}}}{y}\right](1 / 4 t)$, where $L_{y^{2}}^{-1}$ is the Laplace inverse transform with respect to $y^{2}$.

ii) $\left(-\frac{\partial}{2 \pi \sinh \rho \partial \rho}\right) K_{n}^{\mathbb{I H}}\left(t, \rho\left(w, w^{\prime}\right)\right)=K_{n+2}^{\mathbb{I H}}\left(t, \rho\left(w, w^{\prime}\right)\right)$,

iii) $\int_{r}^{\infty} \frac{K_{n+1}^{\mathbb{H}}(t, \rho)}{\sqrt{\cosh ^{2} \rho / 2-\cosh ^{2} r / 2}} \sinh \rho d \rho=K_{n}^{I H}(t, r)$.

Proof. To prove i) use the subordination formula (Strichartz [17], p. 50)

$$
\frac{e^{-y \lambda}}{y}=\frac{1}{\sqrt{\pi}} \int_{0}^{\infty} e^{-u y^{2}} u^{-1 / 2} e^{-\lambda^{2} / 4 u} d u,
$$

or $\frac{\sqrt{\pi} e^{-y \lambda}}{y}=L\left(u^{-1 / 2} e^{-\lambda^{2} / 4 u}\right)\left(y^{2}\right)$, where $(L f)(p)$ is the Laplace transform, and

$$
e^{\frac{-\lambda^{2}}{4 u}}=u^{1 / 2} L_{y^{2}}^{-1}\left(\sqrt{\pi} \frac{e^{-y \lambda}}{y}\right)(u) .
$$

Set $\lambda=\sqrt{\mathcal{L}_{n}}$ and $\frac{1}{4 u}=t$ in the last formula we can write

$$
e^{t \mathcal{L}_{n}}=(4 t)^{-1 / 2} L_{y^{2}}^{-1}\left[\frac{\sqrt{\pi} e^{-y \sqrt{-\mathcal{L}_{n}}}}{y}\right](1 / 4 t),
$$

where $L^{-1}$ is the inverse Laplace transform.

The parts ii) and iii) are consequence of i) and Proposition 4.

Theorem 6. The heat Cauchy problem on hyperbolic space (3) has the unique solution given by

$$
V(t, w)=\int_{H} K_{n}\left(t, w, w^{\prime}\right) V_{0}\left(w^{\prime}\right) d u\left(w^{\prime}\right),
$$

with

$$
K_{n}\left(t, w, w^{\prime}\right)=\frac{\Gamma((n+1) / 2)}{2^{(n+1) / 2} \pi^{n / 2} t^{1 / 2}}
$$




$$
\times \int_{\sigma-i \infty}^{\sigma+i \infty} \frac{\exp \left(\frac{y^{2}}{4 t}\right) \sin y}{\left(\cosh \rho\left(w, w^{\prime}\right)-\cos y\right)^{(n+1) / 2}} d y
$$

Proof. From (7) we see the formula (8) and the proof of Theorem 6 is finished.

Corollary 7. (Davies-Mandouvalos [4] and Lohoue and Rychener [10]) Let $K_{n}\left(t, w, w^{\prime}\right)$ be the heat kernel on the hyperbolic space $\mathbb{H}^{n}$ then we have i) For $n$ odd $K_{n}\left(t, w, w^{\prime}\right)=\left(-\frac{\partial}{2 \pi \sinh \rho \partial \rho}\right)^{\frac{n-1}{2}} \frac{e^{\frac{-\rho^{2}}{t}}}{(4 \pi t)^{1 / 2}}$,

ii) For $n$ even $K_{n}\left(t, w, w^{\prime}\right)=$ $\left(-\frac{\partial}{2 \pi \sinh \rho \partial \rho}\right)^{\frac{n-2}{2}} \int_{\rho}^{\infty}\left(\cosh ^{2} s / 2-\cosh ^{2} \rho / 2\right)^{-1 / 2} \frac{e^{\frac{-s^{2}}{4 t}}}{(4 \pi t)^{3 / 2}} s d s$.

Proof. Set $\cos y=z$,

$$
K_{n}\left(t, w, w^{\prime}\right)=\frac{\Gamma((n+1) / 2)}{2^{(n+1) / 2} \pi^{n / 2} t^{1 / 2}} \int_{\sigma-i \infty}^{\sigma+i \infty} \frac{e^{\frac{(\arccos z)^{2}}{4 t}}}{\left(\cosh \rho\left(w, w^{\prime}\right)-z\right)^{(n+1) / 2}} d z .
$$

To prove the first statment i) we have

$$
K_{1}\left(t, w, w^{\prime}\right)=\frac{1}{2 \pi^{1 / 2} t^{1 / 2}} \int_{\sigma-i \infty}^{\sigma+i \infty} \frac{e^{\frac{(\arccos z)^{2}}{4 t}}}{\left(\cosh \rho\left(w, w^{\prime}\right)-z\right)} d z,
$$

that is

$$
K_{1}\left(t, w, w^{\prime}\right)=\frac{1}{2 \pi^{1 / 2} t^{1 / 2}} \operatorname{Res}_{z=\cosh \rho}\left[\frac{e^{\frac{(\arccos z)^{2}}{4 t}}}{\left(\cosh \rho\left(w, w^{\prime}\right)-z\right)}\right],
$$

and

using ii) of 5 we have i).

$$
K_{1}\left(t, w, w^{\prime}\right)=\frac{1}{\sqrt{4 \pi t}} e^{-\frac{\rho^{2}}{4 t}},
$$

To prove iii), we can write

$$
K_{3}\left(t, w, w^{\prime}\right)=\frac{1}{2^{2} \pi^{3 / 2} t^{1 / 2}} \int_{\sigma-i \infty}^{\sigma+i \infty} \frac{e^{\frac{(\arccos z)^{2}}{4 t}}}{\left(\cosh \rho\left(w, w^{\prime}\right)-z\right)^{2}} d z
$$

and

$$
K_{3}\left(t, w, w^{\prime}\right)=\frac{1}{2^{2} \pi^{3 / 2} t^{1 / 2}} \operatorname{Res}_{z=\cosh \rho}\left[\frac{e^{\frac{(\arccos z)^{2}}{4 t}}}{\left(\cosh \rho\left(w, w^{\prime}\right)-z\right)^{2}}\right],
$$


this gives

$$
K_{3}\left(t, w, w^{\prime}\right)=\frac{1}{2^{2} \pi^{3 / 2} t^{1 / 2}} z \lim _{z \rightarrow \cosh \rho} \frac{d}{d z}\left[e^{\frac{(\arccos z)^{2}}{4 t}}\right]
$$

and finally

$$
K_{3}\left(t, w, w^{\prime}\right)=\frac{1}{(4 \pi t)^{3 / 2}} \frac{\rho}{\sinh \rho} e^{\frac{-\rho^{2}}{4 t}},
$$

using iii) of Theorem 5 we have

$$
K_{2}\left(t, w, w^{\prime}\right)=\int_{\rho}^{\infty}\left(\cosh ^{2} s / 2-\cosh ^{2} \rho / 2\right)^{-1 / 2} \frac{e^{\frac{-s^{2}}{4 t}}}{(4 \pi t)^{3 / 2}} s d s,
$$

Combining (9) and the part i) of Proposition 5 we obtain ii) and the proof of Corollary 8 is finished.

Note that the wave equation on hyperbolic space is studied in Intissar-Ould Moustapha, [6], Bunk et al. [3], Lax-Phillips [8].

\section{Heat kernel for the Bessel operator}

Proposition 8. i) The modified Laplace-Beltrami operator $\mathcal{L}_{2}$ on the hyperbolic space and Bessel operator $L^{a}$ on $\mathbb{R}^{+}$are connected via the formulas

$$
\mathcal{F}_{x_{1}}\left[x_{2}^{-1 / 2} \mathcal{L}_{2} x_{2}^{1 / 2} \Phi\right]\left(a, x_{2}\right)=L^{a}(\mathcal{F} \Phi)\left(\lambda, x_{2}\right),
$$

where the Fourier transform is given by

$$
[\mathcal{F} f](\xi)=\frac{1}{\sqrt{2 \pi}} \int_{\mathbb{R}} e^{-i x \xi} f(x) d x .
$$

ii) The heat kernels for Bessel operator $H^{a}\left(t, x_{2}, x_{2}^{\prime}\right)$ is connected to the heat kernel on the hyperbolic half plane $H_{2}\left(t, z, z^{\prime}\right)$ via the formula

$$
H_{a}\left(t, x_{2}, x_{2}^{\prime}\right)=\frac{1}{\sqrt{x_{2} x_{2}^{\prime}}} \int_{-\infty}^{\infty} e^{-i a\left(x_{1}-x_{1}^{\prime}\right)} H_{2}\left(t, z, z^{\prime}\right) d\left(x_{1}-x_{1}^{\prime}\right) .
$$

Proof. The proof of this proposition is simple and therefore it is left to the reader. 
Theorem 9. The heat Cauchy problem (4) for the Bessel operator $L^{a}$ has the unique solution given by

$$
u(t, x)=\int_{\mathbb{R}} K_{a}\left(t, x, x^{\prime}\right) u_{0}\left(x^{\prime}\right) d x^{\prime},
$$

with

$$
\begin{gathered}
K_{a}\left(t, x, x^{\prime}\right)=\frac{1}{4 \sqrt{\pi} t^{3 / 2}} \\
\times \int_{\cosh ^{2} s / 2 \geq \frac{x^{2}+x^{\prime 2}}{4 x x^{\prime}}}^{\infty} s e^{\frac{-s^{2}}{4 t}} J_{0}\left(|a| \sqrt{4 x x^{\prime} \cosh ^{2} s / 2-x^{2}-x^{\prime 2}}\right) d s .
\end{gathered}
$$

Proof. The proof of this theorem follows from Proposition 8, the Fubini theorem and the formula (Lebedev [9], p.114),

$J_{0}(z)=\frac{1}{[\Gamma(1 / 2)]^{2}} \int_{-1}^{1}\left(1-t^{2}\right)^{\frac{-1}{2}} \cos z t d t=\frac{1}{\pi} \int_{-1}^{1}\left(1-t^{2}\right)^{\frac{-1}{2}} e^{-i z t} t d t$.

\section{Applications}

In this section we give some applications of our results. As an application of Theorem 1 and 9 we give the following corollary giving explicit solution to the Poisson and heat problems with Morse potential Morse [12]. For recent work on Morse potential, the reader can consult (Abdelhaye et al. [1], Ikeda-Matsumoto [5] and Ould Moustapha [13]).

Corollary 10. For $a \in \mathbb{R}$ the problem

$$
\left\{\begin{array}{c}
M^{a} \tilde{u}(y, X)=-\frac{\partial^{2}}{\partial y^{2}} \tilde{u}(y, X),(y, X) \in \mathbb{R}^{+} \times \mathbb{R} \\
\tilde{u}(0, X)=\tilde{u}(X)_{0}, U_{0} \in C_{0}^{\infty}\left(\mathbb{R}^{+}\right)
\end{array}\right.
$$

has the solution given by

$$
\tilde{u}(y, X)=\int_{0}^{\infty} P_{a}\left(y, X, X^{\prime}\right) \tilde{u}_{0}\left(X^{\prime}\right) d X^{\prime},
$$

with

$$
\tilde{P}_{a}\left(y, X, X^{\prime}\right)=\frac{|a|}{\pi} \frac{e^{X+X^{\prime}} \sin y K_{1}\left(|a| \sqrt{\sinh ^{2} \frac{\left(X-X^{\prime}\right)}{2}+\sin ^{2} y / 2}\right)}{\sqrt{\sinh ^{2} \frac{\left(X-X^{\prime}\right)}{2}+\sin ^{2} y / 2}},
$$

where $K_{1}$ is the modified Bessel functions of second kind. 
Proof. Set $X=\ln x$, the problem (10) is transformed into the problem (1) and it is not hard to see the result of theorem from 1.

Theorem 11. The heat Cauchy problem with Morse Potential

$$
\left\{\begin{array}{c}
M^{a} \tilde{V}(t, X)=\frac{\partial}{\partial t} \tilde{V}(t, X),(t, X) \in \mathbb{R}^{+} \times \mathbb{R} \\
\tilde{V}(t, X)=\tilde{V}(X)_{0}, \tilde{V}_{0} \in C_{0}^{\infty}\left(\mathbb{R}^{+}\right)
\end{array},\right.
$$

has the unique solution given by

$$
\tilde{V}(t, X)=\int_{R} K_{a}\left(t, X, X^{\prime}\right) \tilde{V}_{0}\left(X^{\prime}\right) d X^{\prime},
$$

with

$$
\begin{gathered}
K_{a}\left(t, X, X^{\prime}\right)=\frac{1}{4 \sqrt{\pi} t^{3 / 2}} \\
\times \int_{\left|X-X^{\prime}\right|}^{\infty} s e^{\frac{-s^{2}}{4 t}} J_{0}\left(2|a| e^{\left(X+X^{\prime}\right) / 2} \sqrt{\cosh ^{2} s / 2-\cosh ^{2}\left(\left(X-X^{\prime}\right) / 2\right)}\right) d s .
\end{gathered}
$$

Proof. Set $X=\ln x$ the problem (11) is transformed into the problem (4) and it is not hard to see the result of theorem from Theorem 9 .

Remark 12. The Poisson and heat semigroups of the operator of Bessel type

$$
L_{\alpha}=x^{2} \frac{d^{2}}{d x^{2}}+(2 \alpha+3) \frac{d}{d x}+x^{2}+(\alpha+1)^{2}
$$

are considered in Betancor et al. [2].

It is not hard to see that

$$
x^{\alpha+1} L_{\alpha} x^{-\alpha-1}=L^{-i}=x^{2} \frac{\partial^{2}}{\partial x^{2}}+x \frac{\partial}{\partial x}+x^{2} .
$$

Corollary 13. If $a \in i \mathbb{R}^{*}$ and $a=i b$, the problem (1) has the solution given by

$$
v(y, x)=\int_{0}^{\infty} q_{b}\left(y, x, x^{\prime}\right) v_{0}\left(x^{\prime}\right) \frac{d x^{\prime}}{x^{\prime}},
$$

with

$$
q_{b}\left(y, x, x^{\prime}\right)=\frac{-1}{2}|b| \frac{x x^{\prime} \sin y H_{1}^{(1)}\left(|b| \sqrt{x^{2}+x^{\prime 2}-2 x x^{\prime} \cos y}\right)}{\sqrt{x^{2}+x^{\prime 2}-2 x x^{\prime} \cos y}}
$$




$$
q_{b}\left(y, x, x^{\prime}\right)=\frac{-1}{2} \frac{\partial}{\partial y} H_{0}^{(1)}\left(|b| \sqrt{x^{2}+x^{\prime 2}-2 x x^{\prime} \cos y}\right),
$$

wih $H_{1}^{(1)}, H_{0}^{(1)}$ are the Bessel function of the third kind.

Proof. By formula $K_{\nu}\left(z e^{\frac{-i \pi \nu}{2}}\right)=1 / 2 i \pi e^{\frac{i \pi \nu}{2}} H_{\nu}^{(n)}(z)$ (Magnus et al. [11], p.67).

The last application of our result is the explicit formula of the Poisson semigroup on the sphere $S^{n}$.

Corollary 14. The Poisson equation in the sphere $S^{n}$ has the unique solution given by

$$
u(y, \omega)=\int_{S^{n}} P_{n}^{S}\left(y, \omega, \omega^{\prime}\right) u_{0}\left(\omega^{\prime}\right) d \mu\left(\omega^{\prime}\right),
$$

with

$$
P_{n}^{S}\left(y, \omega, \omega^{\prime}\right)=\frac{\Gamma((n+1) / 2)}{\pi^{(n+1) / 2}} \frac{\sinh y}{\left(2 \cosh y-2 \cos d\left(\omega, \omega^{\prime}\right)\right)^{(n+1) / 2}}
$$

Proof. By comparing the radial parts of the Laplace Beltrami operators on the spaces $\mathbb{H}^{n}$ and $S^{n}$ given respectively by

$$
\Delta^{\mathbb{I} H^{n}}=\frac{\partial^{2}}{\partial r^{2}}+(n-1) \operatorname{coth} r \frac{\partial}{\partial r}+\left(\frac{n-1}{2}\right)^{2}
$$

and

$$
\Delta^{S^{n}}=\frac{\partial^{2}}{\partial r^{2}}+(n-1) \cot r \frac{\partial}{\partial r}-\left(\frac{n-1}{2}\right)^{2},
$$

Corollary 14 can be seen from Theorem 3 by an argument of analytic continuation.

Note that the result of this corollary agrees with the formula (4.9) in Taylor [18], p.114.

\section{References}

[1] Y. Abdelhaye, M. Badahi , M.V. Ould Moustapha, Wave kernel for the Schrödinger operator with the Morse potential and applications, F. J. Math. Sci., 102 (2017), 1523-1532. 
[2] J. Betancor, O. Ciaurri, T. Martnez, M. Perez, J.L. Torrea, J.L. Varona, Heat and poisson semigroups for Fourier-Neumann expansions, J. Semi. Forum., 73 (2006), 129142.

[3] U. Bunke, M. Olbrich, A. Juhl, The wave kernel for the Laplacian on locally symmetric spaces of rank one, Theta functions, Trace formulas and the Selberg zeta function, Ann. Global Anal. Geom., 12 (1994), 357-405.

[4] E.B. Davies, N. Mandouvalos, Heat kernel bounds on hyperbolic space and Kleinian groups Proc. London Math. Soc., 3 (1988), 182-208.

[5] N. Ikeda, H. Matsumoto, Brownian motion one the hyperbolic plane and Selberg trace formula, J. Funct. Anal., 163 (1999), 63-110.

[6] A. Intissar, M. V. Ould Moustapha, Solution explicite de l'équation des ondes dans un espace symétrique de type non compact de rang 1, C. R. Acad. Sci. Paris., 321 (1995), 77-81.

[7] I. Cardoso, On the pointwise convergence to initial data of heat and Poisson problems for the Bessel operator, J. Evol. Equ., 17 (2017), 953977.

[8] R.P. Lax, R.S. Phillips, The asymptotic distibution of lattice points in Euclidean and non Euclidean spaces, J. Funct. Anal., 46 (1982), 280-350.

[9] N.N. Lebedev, Special Functions and Their Applications, Dover Publications INC, New York (1972).

[10] N. Lohoue, Th. Rychener, Die Resolvente von auf symmetrischen Räumen vom nichtkompakten, Typ. Comment. Math. Helv., 57 (1982), 445-468.

[11] W. Magnus, F. Oberhettinger, R.P. Soni, Formulas and Theorems for Special Functions of Mathematical Physics, Springer-Verlag Berlin Heidelberg, New York (1966).

[12] P.M. Morse, Diatomic molecules according to the wave mechanics. II. Vibrational levels, Phys. Rev., 34 (1929), 57-64.

[13] M.V. Ould Moustapha, Wave kernel with magnetic field on the hyperbolic plane and with the Morse potential on the real line, Quantum Stud. Math. Found., 7 (2020), 65-75.

[14] A.P. Prudnikov, Yu. A. Brychkov, O.I. Marichev, Special Functions, Integrals and Series, CRC Press, New York (1986). 
[15] S. Keles, S. Bayrakci, Square-like functions generated by the Laplace-Bessel differential operator, Adv. Diff. Equ., 281 (2014), 1687-1847.

[16] E.M. Stein, Singular Integrals and Differentiability Properties of Functions, Princeton University Press, New Jersey (1970).

[17] R. Strichartz, A Guide to Distribution Theory and Fourier Transforms, CRC Press, Boca Racon, Ann Arbor, London, Tokyo (1993).

[18] M. Taylor, Partial Differential Equations II, Springer, Applied Mathematics in Sciences No.116, Second Edition (2011). 